\title{
Prediction method for Surface Roughness based on Cutting parameters and Cloud model reasoning
}

\author{
Weigang Ding ${ }^{1, a}$, Yanchao Yin ${ }^{1, b}$ \\ ${ }^{1}$ Faculty of Mechanical and Electrical Engineering, Kunming University of Science and Technology, \\ Kunming, Yunnan 650500, China \\ aemail: dingweigang@126.com, bemail: yinyc@163.com
}

Keywords: Roughness; Cutting parameters; Precision prediction; Cloud model reasoning;

\begin{abstract}
According to the problem of lower prediction accuracy and narrower prediction range for the common prediction method, a new method for surface roughness prediction is putted forward. on the basis of analyzing large simple data, and cloud reasoning prediction model was established. Then the accuracy surface roughness prediction was realized with this model. Finally, experimental results showed that the presented method is more accurate than other methods in the same conditions.
\end{abstract}

\section{Introduction}

Surface roughness is an important index to measure the quality of products, the surface roughness values should be consistent with the fatigue strength, the performance for the corrosion resistance, accuracy, tribology and aesthetic was required of the qualified products[1], so the surface roughness of the artifact was predicted to optimize the cutting parameters, which is very important to control the quality of the artifact. In recent years, domestic and foreign scholars has made many explorations for roughness prediction methods, which usually adopts the Fuzzy Model[2], Artificial Neural Network Model[3], Genetic Model[4], neural network and fuzzy network combination model, the model of support vector machine and so on. However these models still exists many deficiency, such as the great influence on the prediction accuracy for model structure and parameters selection, at the same time its generalization ability is not strong, the accuracy is not high.

The cloud model was put forward by Chinese scholar Professor Deyi Li[5]based on fuzzy set theory, probability and statistics in the tradition, which is a kind of qualitative and quantitative uncertainty conversion model. According to the information of limited samples, use the multidimensional rules of qualitative reasoning method to predict the roughness of products. At the same time the sample is predicted, the better prediction effect of this method is verified.

\section{Description of Cloud Model for the Roughness Prediction}

The Concept of the Cloud Cloud model is the uncertainty of model transformation between qualitative and quantitative. It through the expectation (Ex), entropy (En) and hyper entropy (He) 3 digital characteristics to describe the qualitative concept of the fuzziness and randomness. Define $U$ is a $U=\{x\}$ domain, $T$ is associated with the $U$ language value. $X$ is an element of $U, X$ for the expression of qualitative concept $\mathrm{T}$ called membership degree $C_{T}(x)$ (or called compatibility or $\mathrm{X}$ and $\mathrm{T}$ ) is a random number with a stable tendency, membership in the domain distribution called membership cloud, referred to as the cloud [13].

Definition: Containing three dimension of the set $U=\left\{x_{1}, x_{2}, x_{3}\right\}$ is called the domain. For the fuzzy sets of the three-dimensional universe $U$ is $A=\left(\mu_{A}\left(x_{1}\right), \mu_{A}\left(x_{2}\right), \mu_{A}\left(x_{3}\right)\right)$, in which $\mu_{A}\left(x_{1}\right)$, $\mu_{A}\left(x_{2}\right), \mu_{A}\left(x_{3}\right)$ for every arbitrary elements $x_{1}, x_{2}, x_{3}$ to the fuzzy set A generated a stable tendency of the random number, so it will be referred to as elements $x_{1}, x_{2}, x_{3}$ to the membership degree of the fuzzy set $\mathrm{A}$. If each element given the $3 \mathrm{D}$ in the domain of expectation, entropy and 
excess entropy, then each element can be generated in a cloud model, the three one dimension cloud model are combined to form a three-dimensional cloud.

Surface Roughness Prediction Model Based on Three-dimensional Multi Rules Because the cutting speed, feed rate and cutting depth is the effect of surface roughness of main process parameters, the cutting speed is divided into four language description level called "bigger, big, general, small ", Feed rate is divided into three language description level called "big, general, small" cutting depth is divided into three language description level called "big, general, small", roughness is divided into six language description level called "biggest bigger, big, general, small, smaller". Try to use cluster analysis method in MATLAB software to deal with 400 samples of data existed, then obtained six cloud model qualitative reasoning rules (see Table 1).

Rule 1: IF cutting speed is bigger AND feed rate is small AND the cutting depth is small, THEN the roughness is smaller

Rule 2: IF cutting speed is bigger AND feed rate is small AND the cutting depth is big, THEN the roughness is small

Rule 6: IF cutting speed is smaller AND feed rate is big AND the cutting depth is general, THEN the roughness is biggest

The above rules mainly displayed as fuzzy concept of qualitative linguistic value, the roughness of the characteristic parameters of cloud object after treatment as shown in table 1. Figure 1 is the cloud model roughness category diagram.

Table 1 Six regulation parameter table

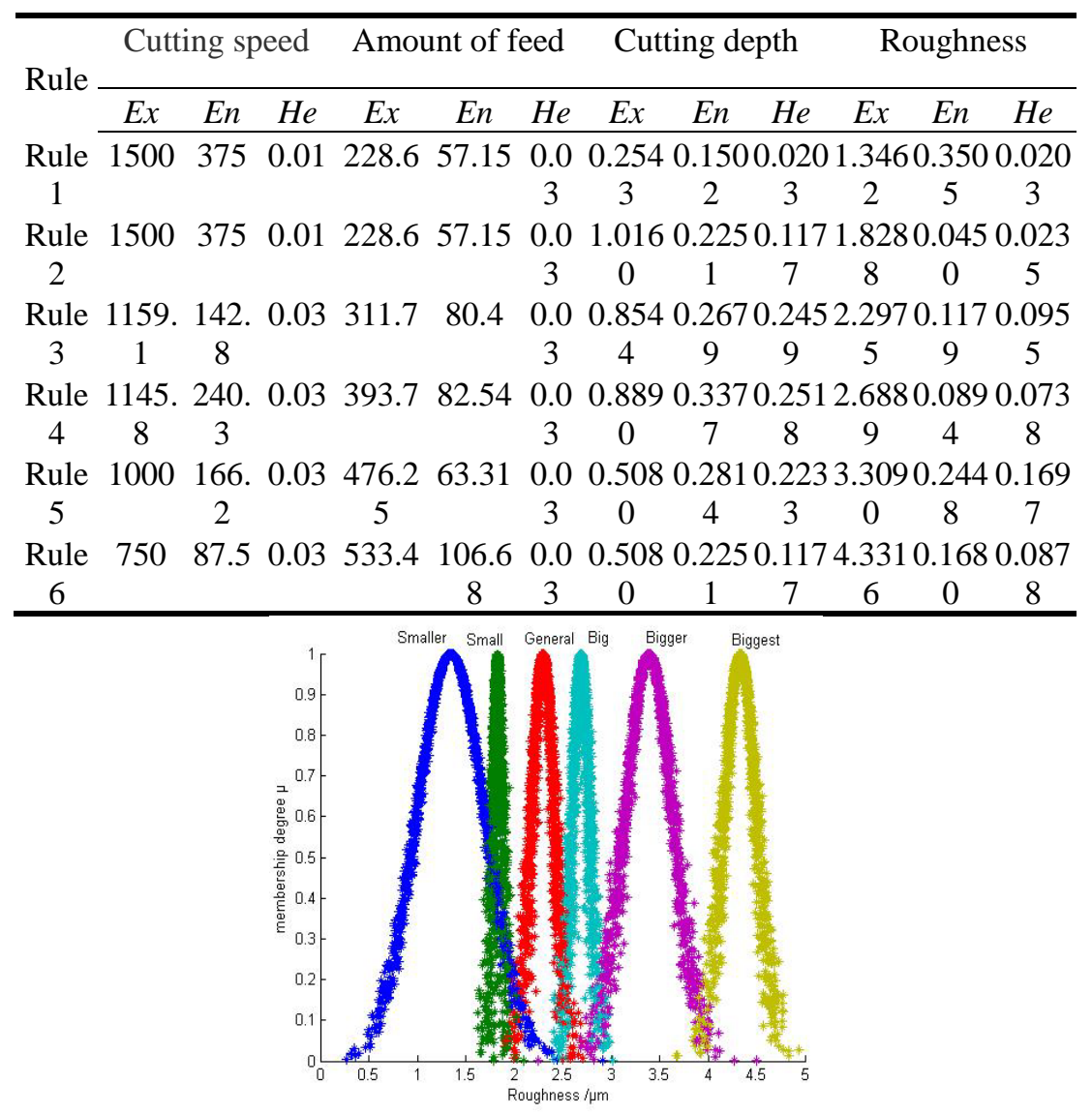

Figure 1 describes the cloud model roughness categories

\section{Roughness Prediction Based on Multidimensional \& Multi-rules Cloud Reasoning Algorithm}

The cloud model rule generator was constructed based on X condition and Y condition of cloud model. Roughness prediction generator based on multidimensional rules cloud model qualitative reasoning was created by a plurality of n-dimensional X conditional clouds generator and a plurality 
of one-dimensional Y conditional clouds generator.

According to the rules extracted from the data, under the given conditions roughness can be predicted. Roughness prediction index of the artifact have cutting speed, feed rate, cut depth and so on. Specific multidimensional cloud model based on the rules of qualitative reasoning process is as follows:

Algorithm: multidimensional rules of qualitative reasoning of cloud model algorithm

Step 1: A one-dimensional normal random number $E n_{b n i}{ }^{\prime}$, expected value is $E n_{b n i}$, the standard deviation is $\mathrm{He}_{b n i}$, then according to the given condition value, calculate the membership of $\mu$ :

$$
\mu=e^{-\left(\frac{\left(x_{1}-E x_{b 11}\right)^{2}}{2 E n_{b 11}^{2}}+\frac{\left(x_{2}-E x_{b 21}\right)^{2}}{2 E n_{b 21}^{2}}+\ldots+\frac{\left(x_{n}-E x_{b n}\right)^{2}}{2 E n_{b n 1}^{2}}\right)}
$$

Step 2.1: If activate a rule, then according to the obtained roughness prediction knowledge rules, produced by $E n_{U 1 i}$ as the expected value, $H e_{U 1 i}$ as the standard deviation of the n-dimensional normal value of $E n_{U 1 i}$, then calculate $y_{i}$ :

$$
y_{i}=E_{U 1 i} \pm \sqrt{-2 \ln (\mu)} E n_{U 1 i}^{\prime}
$$

Step 2.2: Define $\left(y_{i}, \mu\right)$ is a cloud. Return to step 2.1, repeated several times, the average value of expected value can be used as output.

Step 3.1: If activate the two or more than two rules. Generate $\mu_{i}$, define $\mu_{1}$ is the maximum, $\mu_{2}$ is the second. The two corresponding single rules, according to the given consequents ( $\left.E n_{U 11}, H e_{U 11}\right)$, randomly generated with $E n_{U 11}$ as expected value, $H e_{U 11}$ as variance of the one-dimensional normal random value $E n_{U 111}, E n_{U 112}$; According to the following formula, anti-computing two $y_{1}$, under the conditions of $\mu_{1}, E n_{U 111}$, anti-computing two $y_{2}$, under the conditions of $\mu_{2}, E n_{U 112}$ :

$$
\mu_{1}=e^{-\frac{\left(y_{1}-E x_{U 11}\right)^{2}}{2 E n_{U 111}^{2}}}, \mu_{2}=e^{-\frac{\left(y_{2}-E x_{U 11}\right)^{2}}{2 E n_{U 112}^{2}}}
$$

Step 3.2: Select two outermost cloud droplets $\left(y_{1}, \mu_{1}\right)$ and $\left(y_{2}, \mu_{2}\right)$, to construct a virtual concept with geometric method. The digital characteristics of the virtual cloud as ( $E x, E n, H e$ ), a provisional hyper entropy $H e=0$, through the geometric method for solving the variance components available virtual cloud expected value:

$$
E x=\frac{y_{1} \sqrt{-2 \ln \left(\mu_{2}\right)}+y_{2} \sqrt{-2 \ln \left(\mu_{1}\right)}}{\sqrt{-2 \ln \left(\mu_{2}\right)}+\sqrt{-2 \ln \left(\mu_{1}\right)}}
$$

Step 4: Repeat steps 1-3 until the number of values for the surface roughness prediction value to meet the $\mathrm{Y}$ requirements.

\section{Application Examples of Surface Roughness Prediction using Cloud Model}

Experimental conditions: the artifact material is the 6061 aluminum block, machine tool is the CNC vertical machining center, cutting parameters is the four groove diameter of $3 / 4$ high speed steel. Each sample object is described with the cutting speed $x_{1}$, feed rate $x_{2}$, depth of cut $x_{3}$ and machined surface roughness y. Production, $x_{1}=750,1000,1250,1500(\mathrm{r} / \mathrm{min}), x_{2}=152.4,304.8$, 457.2, $609.6(\mathrm{~mm} / \mathrm{min}), \quad x_{3}=0.254,0.762,1.270(\mathrm{~mm}) ; \mathrm{y}$ achieved by Hommel surface roughness instrument.

Apply the multidimensional rules of qualitative reasoning generator to predict 36 validation data on table 2. In table $2 \mathrm{y}$ is the actual value, $\mathrm{y}^{\wedge}$ is the results of prediction. Figure 3 shows the prediction relative error distribution. 
Table2 Validation sample forecast

\begin{tabular}{|c|c|c|c|c|c|c|}
\hline Object & $\begin{array}{c}X_{1} \\
\mathrm{r} / \mathrm{min}\end{array}$ & $\begin{array}{c}x_{2} \\
\mathrm{~mm} / \mathrm{min}\end{array}$ & $\begin{array}{c}x_{3} \\
\mathrm{~mm}\end{array}$ & $\mathrm{y}$ & $\mathrm{y}^{\wedge}$ & Error \\
\hline 1 & 750 & 228.6 & 0.254 & 2.7686 & 2.7587 & 0.4 \\
\hline 2 & 750 & 228.6 & 0.762 & 2.5134 & 2.7118 & 7.9 \\
\hline 3 & 750 & 228.6 & 1.270 & 2.4130 & 2.2588 & 6.4 \\
\hline 4 & 750 & 381.0 & 0.254 & 3.1750 & 3.3043 & 4.1 \\
\hline 5 & 750 & 381.0 & 0.762 & 3.0988 & 2.9691 & 4.2 \\
\hline 6 & 750 & 381.0 & 1.270 & 2.6416 & 2.7207 & 3.0 \\
\hline 7 & 750 & 533.4 & 0.254 & 4.5212 & 4.3321 & 4.2 \\
\hline 8 & 750 & 533.4 & 0.762 & 4.1402 & 4.3445 & 3.9 \\
\hline 9 & 750 & 533.4 & 1.270 & 3.8100 & 3.8203 & 0.3 \\
\hline 10 & 1000 & 228.6 & 0.254 & 2.3368 & 2.2493 & 7.7 \\
\hline 11 & 1000 & 228.6 & 0.762 & 2.4381 & 2.6436 & 8.4 \\
\hline 12 & 1000 & 228.6 & 1.270 & 2.5908 & 2.7211 & 5.0 \\
\hline 13 & 1000 & 381.0 & 0.254 & 3.2766 & 3.0346 & 7.4 \\
\hline 14 & 1000 & 381.0 & 0.762 & 2.7432 & 2.4173 & 11.9 \\
\hline 15 & 1000 & 381.0 & 1.270 & 2.3368 & 2.6823 & 14.8 \\
\hline 16 & 1000 & 533.4 & 0.254 & 3.7846 & 3.8573 & 1.9 \\
\hline 17 & 1000 & 533.4 & 0.762 & 3.6830 & 3.3814 & 8.2 \\
\hline 18 & 1000 & 533.4 & 1.270 & 2.8448 & 2.8085 & 1.3 \\
\hline 19 & 1250 & 228.6 & 0.254 & 2.0320 & 2.1465 & 5.6 \\
\hline 20 & 1250 & 228.6 & 0.762 & 2.0828 & 1.8219 & 12.5 \\
\hline 21 & 1250 & 228.6 & 1.270 & 2.3368 & 2.3920 & 2.4 \\
\hline 22 & 1250 & 381.0 & 0.254 & 2.7178 & 2.4932 & 8.3 \\
\hline
\end{tabular}

\begin{tabular}{|c|c|c|c|c|c|c|}
\hline 23 & 1250 & 381.0 & 0.762 & 2.4638 & 2.4932 & 1.2 \\
\hline 24 & 1250 & 381.0 & 1.270 & 2.2098 & 2.0070 & 9.2 \\
\hline 25 & 1250 & 533.4 & 0.254 & 3.2766 & 3.1581 & 3.6 \\
\hline 26 & 1250 & 533.4 & 0.762 & 2.4892 & 2.4256 & 2.6 \\
\hline 27 & 1250 & 533.4 & 1.270 & 2.6670 & 2.6988 & 1.2 \\
\hline 28 & 1500 & 228.6 & 0.254 & 1.3462 & 1.3462 & 0 \\
\hline 29 & 1500 & 228.6 & 0.762 & 1.8796 & 1.8471 & 1.7 \\
\hline 30 & 1500 & 228.6 & 1.270 & 1.7780 & 1.7969 & 1.1 \\
\hline 31 & 1500 & 381.0 & 0.254 & 2.7940 & 2.7453 & 1.7 \\
\hline 32 & 1500 & 381.0 & 0.762 & 2.1336 & 2.2323 & 4.6 \\
\hline 33 & 1500 & 381.0 & 1.270 & 2.5146 & 2.5146 & 6.9 \\
\hline 34 & 1500 & 533.4 & 0.254 & 3.0226 & 3.0899 & 2.2 \\
\hline 35 & 1500 & 533.4 & 0.762 & 2.5908 & 2.4932 & 3.8 \\
\hline 36 & 1500 & 533.4 & 1.270 & 2.8702 & 2.8020 & 2.4 \\
\hline
\end{tabular}

Table3 Compare with the prediction model error(Training samples 400,Validation sample 36)

\begin{tabular}{cccccccc}
\hline Model & $\begin{array}{c}>25 \\
\%\end{array}$ & $\begin{array}{c}20 \sim 25 \\
\%\end{array}$ & $\begin{array}{c}15 \sim 20 \\
\%\end{array}$ & $\begin{array}{c}10 \sim 15 \\
\%\end{array}$ & $\begin{array}{c}5 \sim 10 \\
\%\end{array}$ & $\begin{array}{c}0 \sim 5 \\
\%\end{array}$ & $\begin{array}{c}\text { Average } \\
\text { Error/\% }\end{array}$ \\
\hline MRA1 $^{[2]}$ & 1 & 3 & 4 & 3 & 10 & 15 & 8.32 \\
MRA2 $^{[3]}$ & 2 & 5 & 3 & 5 & 9 & 12 & 10.74 \\
MRA3 $^{[3]}$ & 1 & 1 & 3 & 9 & 8 & 14 & 8.73 \\
FN1 $^{[2]}$ & 1 & 1 & 1 & 6 & 11 & 16 & 6.89 \\
FN2 $^{[2]}$ & 2 & 4 & 2 & 5 & 12 & 11 & 9.26 \\
KM1 $^{[2]}$ & 1 & 1 & 1 & 6 & 11 & 6 & 6.89 \\
KM2 $^{[2]}$ & 1 & 2 & 3 & 7 & 10 & 13 & 8.74 \\
ANN1 $^{[3]}$ & 1 & 0 & 2 & 9 & 11 & 13 & 7.93 \\
Cloud $_{\text {Model }}$ & 0 & 0 & 0 & 3 & 10 & 23 & 4.88 \\
\hline
\end{tabular}

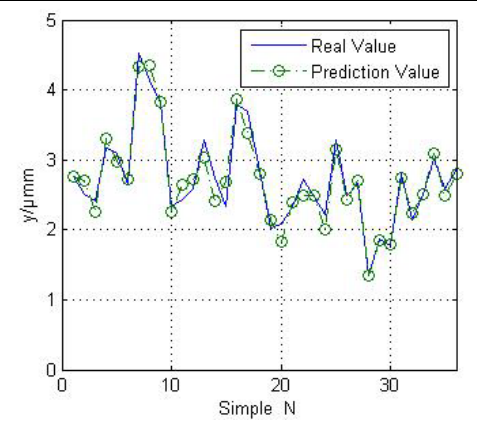

Figure 2 Compare with real value and prediction value

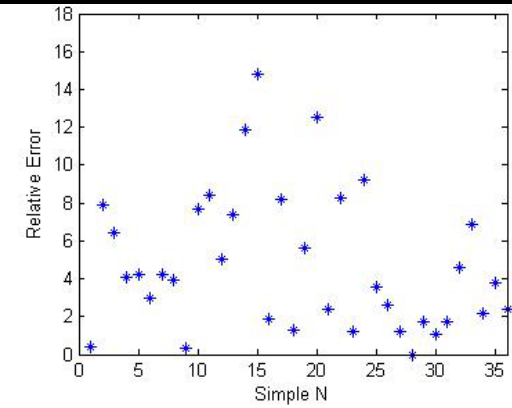

Figure 3 Error distribution

\section{Conclusion}

Prediction method for surface roughness based on cutting parameters and cloud model reasoning was put forwarded, and the uncertainty characteristic roughness prediction was described that will 
be the first time to apply cloud model theory in the mechanical processing. Through the digital characteristics of surface effect on machining roughness factors, the digital features of cloud model was determined. Mix the fuzzy and randomness in together to overcome the membership functions of the fuzzy sets theory inherent defect, a new method for other similar problems was provided in mechanical engineering. In the prediction accuracy, this model has more superiority in dealing with uncertain problems, the experimental results also show that the proposed method has good effects in the aspect of prediction performance and prediction accuracy, has carried on the beneficial exploration to the roughness prediction method of high precision.

\section{Acknowledgement}

In this paper, the research was sponsored by the National Natural Science Foundation of China(51365022) and the Key project of Education Department of Yunnan Province(2014Z032).

\section{References}

[1] VIKAS U, P. K. J, N. K. M, In-process prediction of surface roughness in turning of Ti-6Al-4V alloy using cutting parameters and vibration signals[J]. Measurment, 2013, 46: 154-160.

[2] LOU S. Development of four in-process surface recognition systems to predict surface roughness in end milling[D]. Ames, IA, USA: lowa State University, 1997.

[3] TSAI Y S, CHEN J C, LOU S J. An in-process surface recognition system based on neural networks in end milling cutting operations[J]. Interntional Journal of Machine Tools \& Manufacture, 1999, 39(4): 583-605.

[4] BREZOCHIK M, KOVACIC M, FICKO M. Prediction of surface roughness with genetic

[5] Li Deyi, Liu Changli. Study on the Universality of the Normal Cloud Model[J]. Engineering Science, 2004, 6(8): 30-32. 